\title{
Cervical nerve roots and the dural sheath: a histological study using human fetuses near term
}

\author{
Kei Kitamura ${ }^{1}$, Masahito Yamamoto ${ }^{2}$, Yoshinosuke Hirota ${ }^{2}$, Noriyuki Sato ${ }^{2}$, Toshimasa Machida ${ }^{2}$, \\ Noboru Ishikawa ${ }^{1}$, Hitoshi Yamamoto ${ }^{1}$, Gen Murakami ${ }^{2,3}$, Shinichi Abe ${ }^{2}$ \\ ${ }^{1}$ Department of Histology and Embryology, Tokyo Dental College, Tokyo, ${ }^{2}$ Department of Anatomy, Tokyo Dental College, Tokyo, ${ }^{3}$ Division of Internal \\ Medicine, Jikou-kai Home Visit Clinic, Sapporo, Japan
}

\begin{abstract}
We have previously reported that the thoracolumbar posterior nerve root shows a tortuous epidural course, based on studies of human fetuses near term. For comparison with the cervical nerve, examinations were conducted using frontal, sagittal and horizontal sections of cervical vertebrae from 22 fetuses at 30-38 weeks of gestation. The cervical nerve root showed a short, straight and lateral course near the zygapophysial joint. Multiple rather than single bundles of the cervical posterior root seemed to account for the majority of sensory nerve fibers innervating the upper extremity. Fasciculation of rootlets was evident near the thoracolumbar spinal cord, whereas it was seen in the dural pocket at the nerve exit from the dural sac although both sites were subdural. As in the thoracolumbar region, the nerve sheath was continuous with the dura mater and independently surrounded each of the anterior and posterior roots. Radicular arteries were few in the cervical region. In 2 of the 22 fetuses ( 31 weeks and 33 weeks), there was a segmental, unilateral abnormality of nerve rootlet fasciculation where the dorsal root ganglion was located lateral or peripheral to the intervertebral region. Long nerve roots running inferiorly are a necessary adaptation to the delayed and marked growth of the thoracolumbar vertebral column. In children, the cervical nerve roots are likely to be affected by movement or dislocation of the vertebrae. The segmental abnormality of the cervical nerve root may be linked to rare variations in the brachial plexus.
\end{abstract}

Key words: Spinal nerve, Fetuses, Anatomy

Received April 7, 2020; Revised August 17, 2020; Accepted August 31, 2020

\section{Introduction}

Based on gross observation of 20 adult specimens and histological studies, Tubbs et al. [1] described a region-specific difference in the architecture of the spinal nerve root sheath: the cervical anterior and posterior nerve roots were appar-

\section{Corresponding author:}

Masahito Yamamoto (iD

Department of Anatomy, Tokyo Dental College, 2-9-18 Misaki-cho, Chiyoda-ku, Tokyo 101-0o61, Japan

E-mail:yamamotomasahito@tdc.ac.jp ently separated, and each was surrounded by a dural sheath, in contrast to the common sheath shared by both nerve roots in the thoracolumbar region. They considered the arachnoid to be continuous with a reticular layer along the nerve roots peripheral to the pocket-like protrusion of the dura mater at the nerve exit. In view of the large volume of hard tissues that were likely present, the histological details described were those after dissection, and therefore as a result the topographical anatomy presented might have been altered.

At the thoracolumbar level, frontal sections demonstrate multiple spinal nerve roots with long longitudinal courses through the epidural space $[2,3]$. Using frontal sections from human fetuses near term, we have previously shown that 
the posterior nerve root often has a tortuous epidural course resembling a string of beads at the thoracolumbar level [3], whereas a straight anterior root is usually accompanied by a radicular artery in this region (Fig. 1). The anterior and posterior nerve rootlets run inferolaterally, and as described by Tubbs et al. [1], each of them enters an independent pocketlike protrusion of the dura mater in the thoracolumbar region. In addition, surgeons and neurologists have noted that the dorsal root ganglion (DRG) of the second cervical nerve differs in location from that of the other cervical nerves [4].

Consequently, the aim of the present study was to characterize the cervical region-specific morphology of the spinal nerve roots, focusing on 1) the dural pocket at the nerve exit, 2) fasciculation of nerve rootlets by the sheath, and 3) the topographical relationship between the nerve root and the radicular artery. The sections we employed were prepared from the entire cervical vertebral column, with the intention of clarifying the actual topographical anatomy without dissection-related artifacts.

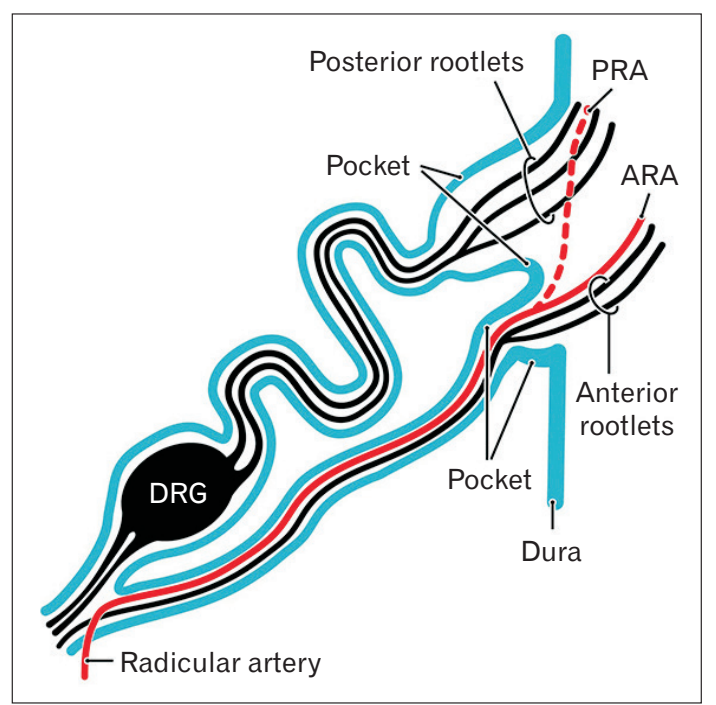

Fig. 1. Diagram of thoracic spinal nerve roots in the human fetus near term. Blue color indicates the dura mater and the nerve sheath continuous with the dura. The posterior and anterior rootlets enter superior and inferior 'pockets' of the dura mater, respectively. The posterior root is characterized by a wavy course in contrast to the straight anterior root. A radicular artery, if present at the nerve level, runs along the anterior root and, in the dural pocket, decides the direction along the posterior or anterior rootlets, i.e., the PRA or ARA. ARA, anterior radicular artery; DRG, dorsal root ganglion; PRA, posterior radicular artery. Modified from Cho et al. Biomed Res Int 2016 Mar 16 [Epub]. http://dx.doi. org/10.1155/2016/8163519 [3].

\section{Materials and Methods}

The study was performed in accordance with the provisions of the Declaration of Helsinki 1995 (as revised in Edinburgh 2000). We examined paraffin-embedded histological sections from 22 fetuses near term (gestational age approximately 30-38 weeks; crown-rump length [CRL] 250-315 $\mathrm{mm})$. All fetuses were part of the large collection kept at the Department of Anatomy, Universidad Complutense, Madrid, and were the products of miscarriages and ectopic pregnancies at the Department of Obstetrics of the University. All sections were stained with hematoxylin and eosin (H\&E). The sectional planes were frontal (3 fetuses), sagittal (12) and horizontal (7). The study was approved by Complutense University ethics committee (B08/374). Observations and photography were usually performed with a Nikon Eclipse 80.

\section{Results}

\section{Observations of frontal sections}

Frontal sections clearly demonstrated a sac-like or baglike appearance of the spinal dura mater that contained the cervical spinal cord (Fig. 2). In the present study we termed this the 'dural sac', although to various extents the dura mater continued to a sheath surrounding the nerve roots and DRG. The sac was thick and reached $0.1 \mathrm{~mm}$ at its thickest part (Fig. 3). The zygapophysial or facet joint was evident near the nerve roots. The joint cavity extended widely from anterior sections partly containing the vertebral body (Fig. $2 \mathrm{~A}, \mathrm{~B})$ to posterior sections including the posterior arch of the first vertebra (Fig. 2I). The epidural veins appeared to provide bilateral trunks, which were crossed or penetrated by the spinal nerve roots.

When the nerve root crossed and exited from the dural $\mathrm{sac}$, its direction changed from inferior to horizontal: the angle at the exit was almost $120^{\circ}$, but sometimes less and nearly $90^{\circ}$ (the anterior root in Fig. 3E). At the nerve exit from the dural sac, a pocket-like protrusion of the dura mater was evident at the lower cervical level (Figs. 2E, G, 3G, I). Higher (second and third) cervical nerve roots exited from the dural sac in more anterior sections (Fig. 2A, B), whereas in posterior sections the fifth and/or sixth nerve roots did so (Fig. $2 \mathrm{~F}-\mathrm{I})$. Sometimes, the anterior and posterior roots crossed the sac together (Fig. 3D, G). The posterior root was usually composed of two to three bundles, in contrast to the anterior root, which was a single bundle (Fig. 3B, E, G). No nerve root 

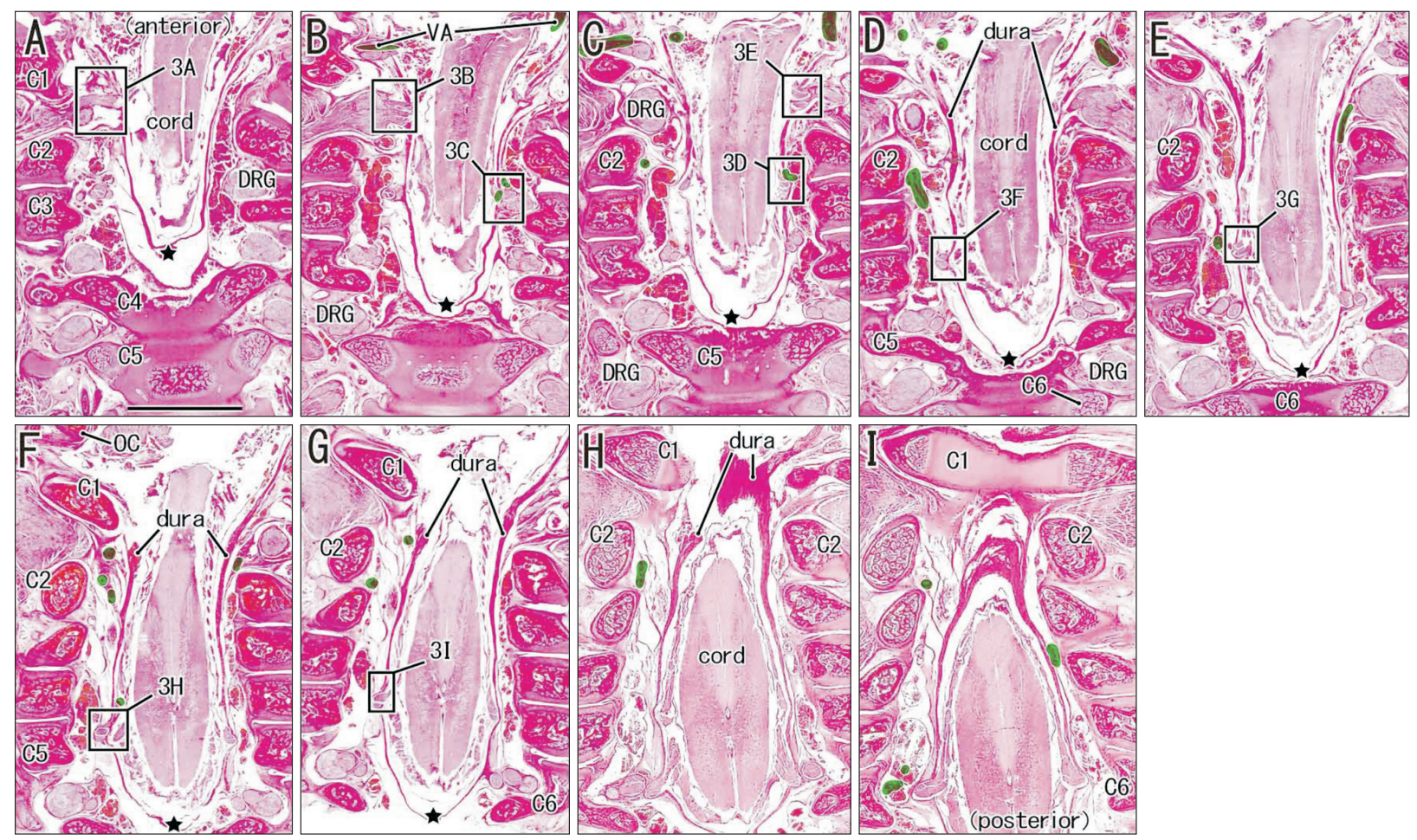

Fig. 2. Frontal sections of the cervical cord and nerve roots from a fetus measuring $270 \mathrm{~mm}(\mathrm{OC} 27)$. (A) displays the most anterior site in the figure, and (I) the most posterior. Green dots and short lines indicate candidate radicular arteries. Stars indicate the bottom of the dural sac. Higher cervical nerve roots cross the dural sac in more anterior sections: the second and third cervical nerves appear in (A, B), whereas the fifth and/or sixth nerves appear in (F-I). Squares in (A-G) (nerve roots) are shown in Fig. 3 at higher magnification. (A-I) were prepared at the same magnification (scale bar in A, $5 \mathrm{~mm}$ ). None of the figures show a radicular artery candidate with a longitudinal course (colored green). C1-C6, first-sixth cervical vertebrae; DRG, dorsal root ganglion; OC, occipital basilar part; VA, vertebral artery.

exhibited a tortuous course after exiting from the dural sac. No radicular artery candidate with a longitudinal course was found in frontal sections. In addition, the DRG of the second cervical nerve was located specifically near the dural sac (Fig. 2C).

\section{Observations of sagittal sections}

Since the nerve roots ran laterally rather than inferiorly in the cervical region, sagittal sections demonstrated nerve rootlet fasciculation more clearly than frontal sections (Figs. $4,5)$. Fasciculation of nerve rootlets occurred in the dural pocket at the point where the nerve exited from the dural sac (Fig. 5A, E). The anterior nerve rootlets were almost always fasciculated into a single bundle at the nerve exit. In contrast, the posterior rootlets were likely to fasciculate into two or three bundles (Figs. 4E-G, 5E, F). However, multiple bundles of the posterior root were surrounded by a common sheath continuous with the dural sac. Irrespective of whether the posterior root was single or multiple, the anterior nerve root was located immediately anterior to the corresponding pos- terior root(s). During its long course, the anterior root was often separated from the other components without intermingling. This allowed us to trace it from the dural pocket (Fig. 5A, E) to its peripheral course along the DRG surface (Fig. 5D, G).

The dural sac pocket at the nerve exit (Fig. 5A, E) was larger than that seen in frontal sections (Fig. 3G, I), and thus it was longer anteroposteriorly than mediolaterally. No nerve root exhibited a tortuous course after exiting the dural sac. However, changes in nerve root direction were difficult to identify in sagittal sections. Epidural veins tended to run along the anterior, rather than posterior, wall of the vertebral canal (Figs. 4F, 5A). A few radicular artery candidates took a longitudinal course along the nerve root (Fig. 5C, G). The zygapophysial joint cavity appeared to extend widely from medial sections containing the spinal cord (Fig. 4E) to lateral sections including the DRG (Fig. 4H).

\section{Observations of horizontal sections}

Horizontal sections clearly demonstrated a topographical 


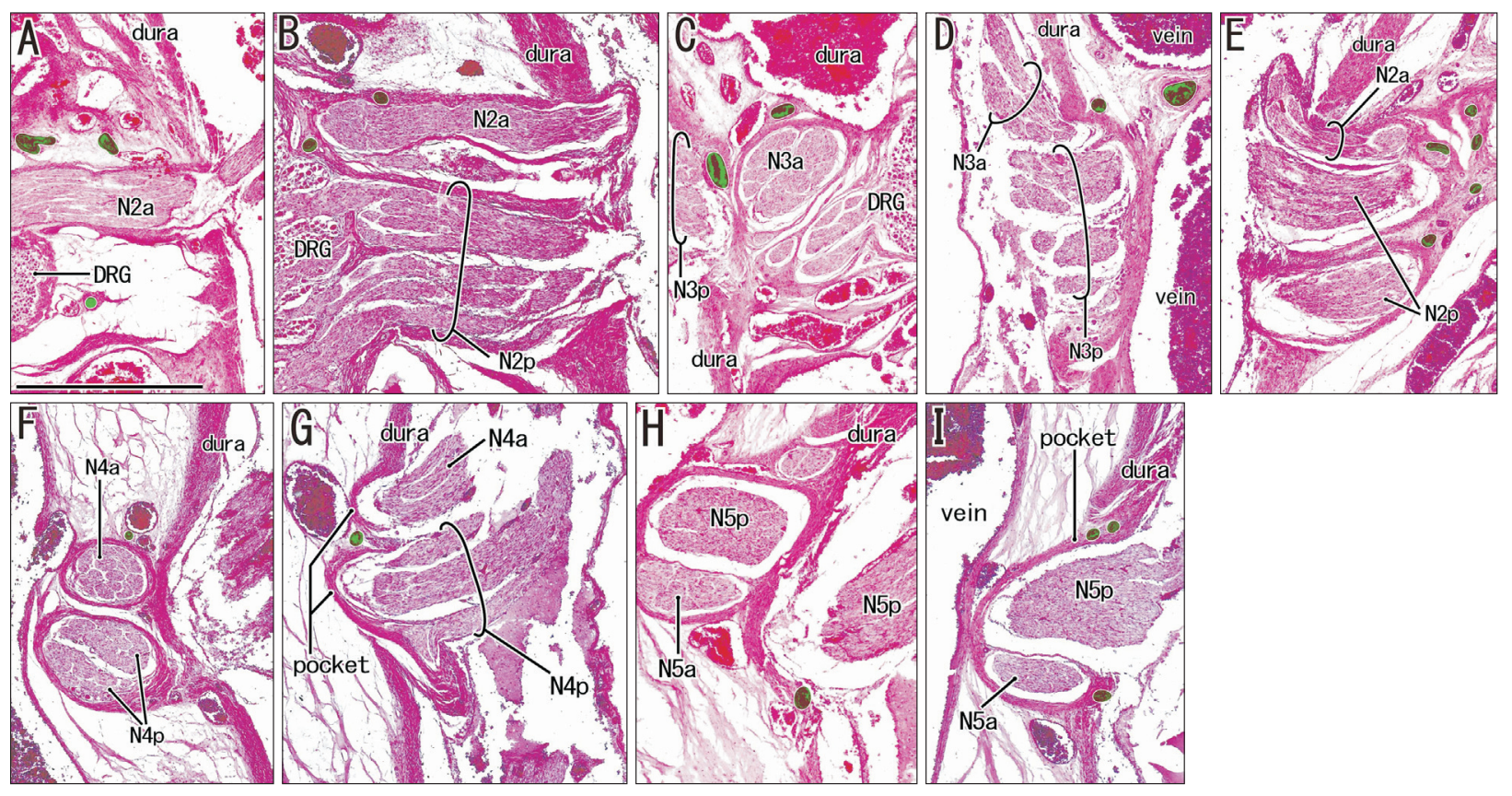

Fig. 3. Cervical nerve roots at the dural sac exit. Higher-magnification views of the squares shown in Fig. 2. Four combinations of figures display the same nerve root: (A, B) second cervical nerve, (C, D) third, (F, G) fourth, and (H, I) fifth. In the second and fifth cervical nerves, the anterior root (N2a in A; N5a in H) crosses the dural sac anterior to the corresponding posterior root (N2p in B; N5p in I). However, in the third and fourth cervical nerves, the anterior and posterior roots cross the sac together (N3a and N3p in D; N4a and N4p in G). The posterior root is usually composed of 2-3 bundles, in contrast to the single bundle of the anterior root. The dural sac forms a pocket-like structure receiving the nerve root (E, G, I). None of the figures show a radicular artery candidate with a longitudinal course (colored green). (A-I) were prepared at the same magnification (scale bar in A, $1 \mathrm{~mm}$ ). DRG, dorsal root ganglion.

relationship between the anterior and posterior nerve roots, the former being anterior to the latter and the two being separated by a narrow space (Fig. 6). Likewise, a continuation of the dura mater was easily traced from the pocket at the nerve exit to the sheath of the DRG. However, neither the nerve root nor its sheath was likely to contain the arachnoid. Moreover, in contrast to frontal and sagittal sections, we often found a radicular artery running along the anterior root (Fig. 6D). The zygapophysial joint and a vertebral arch were seen together in a horizontal section containing the DRG (Fig. 6A-C), the latter being located close to the superior articular process of the lower adjacent vertebra. At the joint, the lower vertebral element was located anteriorly and the upper vertebra posteriorly (Fig. 6C).

\section{Segmental abnormality}

In two of the 22 fetuses examined, a segmental and unilateral abnormality of nerve rootlet fasciculation was observed: the sixth cervical nerve at 33 weeks (CRL $280 \mathrm{~mm}$; Figs. 7,8 ) and the seventh nerve at 31 weeks (CRL $260 \mathrm{~mm}$ ). In these lower cervical nerves, rootlet fasciculation did not occur in the epidural space including the dural pocket but in the interradicular area containing the DRG. However, the fasciculation was loose and did not involve the dura mater. Moreover, the unilateral DRG of the segment was not present between the vertebrae but embedded in the scalenus muscles (Fig. 7I, J). Thus, the single DRG was located far anterolateral to the vertebral artery. In spite of the lack of fasciculation near the dural sac, the anterior and posterior roots were discriminated in lateral sections containing the vertebral artery, each of them being loosely aggregated (Fig. 8D). The vertebrae and radicular artery candidates showed no evident abnormality.

\section{Discussion}

O'Rahilly et al. [5] were the first to examine the whole vertebral column of human fetuses, although the specimens were at relatively early stages ( 8 weeks). Castellana and Kósa [6] demonstrated cervical vertebral growth in late-stage human fetuses, but did not examine the whole column. The present frontal and sagittal sections appear to be the first to demonstrate the topographical anatomy of the whole cervical column, including the cervical nerves, at the late stage, 

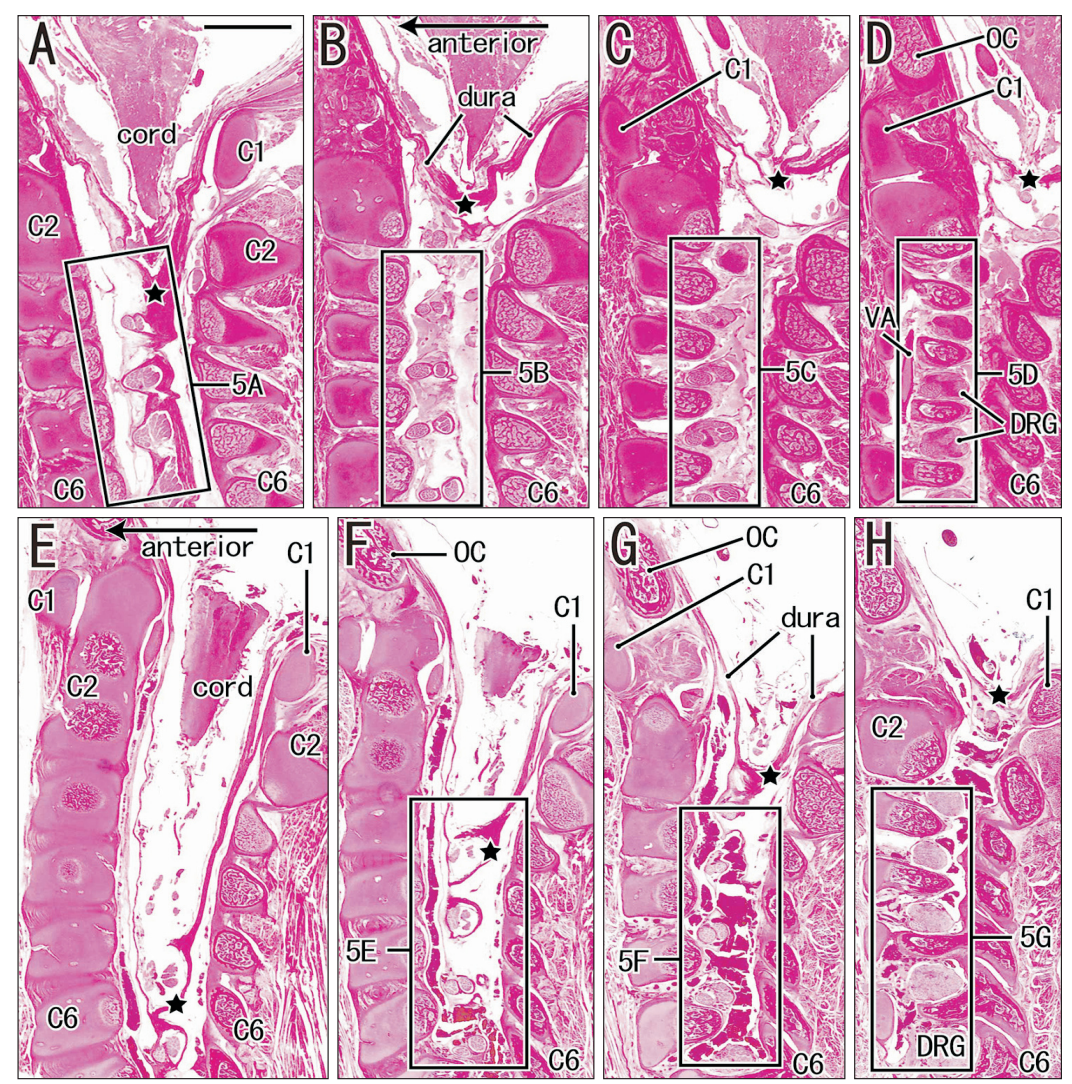

Fig. 4. Sagittal sections of cervical nerve roots from two fetuses near term. (A-D) display sections from a fetus with a CRL of 302 mm (OC26), and (E$\mathrm{H}$ ) those from a fetus with a CRL of $285 \mathrm{~mm}$ (OC28). (A, E) show the most medial site in the specimen, and (D, H) the most lateral. Stars indicate the bottom of the dural sac. The rootlets are bundled into two nerve roots in (B), and into two or three bundles in (F, G) (higher magnification, see Fig. 5). (A-H) were prepared at the same magnification (scale bar in A, $5 \mathrm{~mm}$ ). C1, C2, and C6, the first, second and sixth cervical vertebrae; CRL, crown-rump length; DRG, dorsal root ganglion; OC, occipital basilar part; VA, vertebral artery.
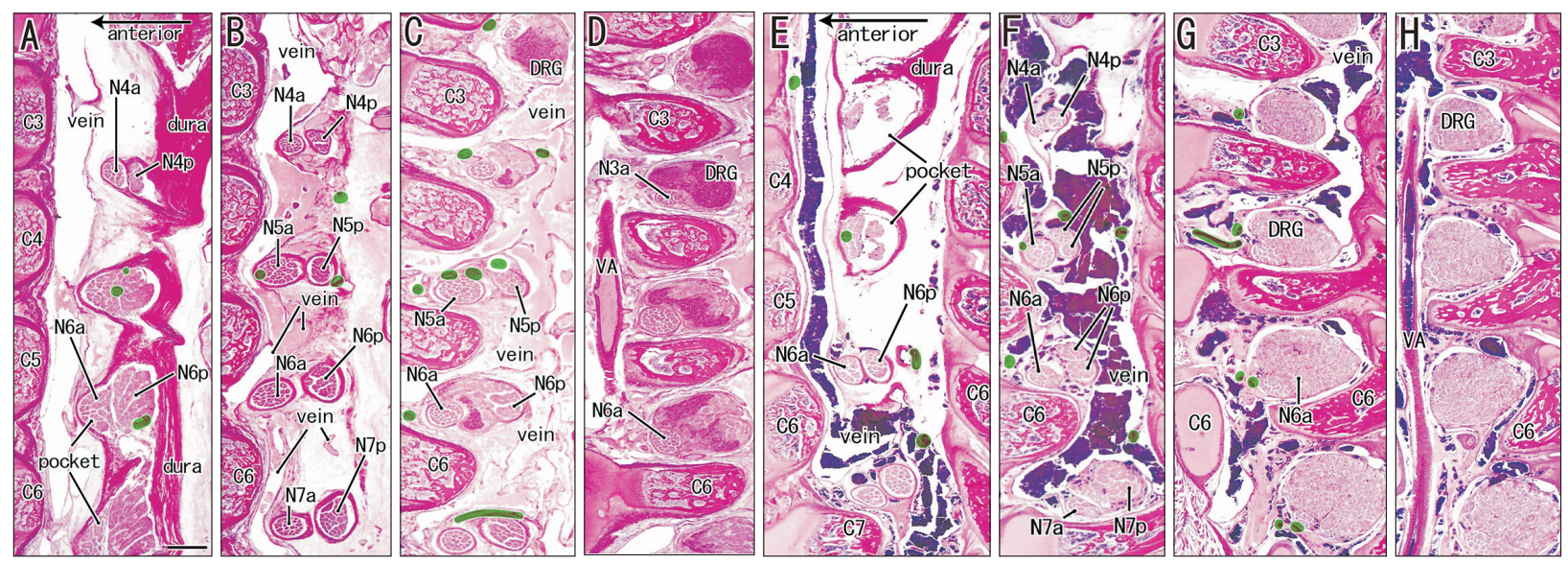

Fig. 5. Sagittal sections showing exit of cervical nerve roots from the dural sac to the DRG. Higher-magnification views of the squares shown in Fig. 4. (A-D) show sections from a fetus with a CRL of $302 \mathrm{~mm}$ (OC26), and (E-H) those from a fetus with a CRL of 285 mm (OC28). (A, E) show the most medial site in the specimen, and $(\mathrm{D}, \mathrm{H})$ the most lateral. The anterior nerve roots of the fourth-seventh cervical nerves $(\mathrm{N} 4 \mathrm{a}-\mathrm{N} 7 \mathrm{a})$ are adjacent to the anterior side of the corresponding posterior roots (N4p-N7p) in (B, F). The DRG is surrounded by veins (G). The anterior root can be clearly identified near and around the ganglion (N6a in D, G). The posterior root of the fifth and sixth cervical nerves (N5P, N6p) is composed of 2-3 bundles in $(\mathrm{F})$, in contrast to a single bundle in $(\mathrm{C})$. The pocket of the dural sac at the nerve exit (A, E) is much larger than that seen in frontal sections (Fig. 3G, I). A few radicular artery candidates (colored green) show a longitudinal course (C, G). (A-H) were prepared at the same magnification (scale bar in A, $1 \mathrm{~mm}$ ). C3-C6, the third-sixth cervical vertebrae; CRL, crown-rump length; VA, vertebral artery. 

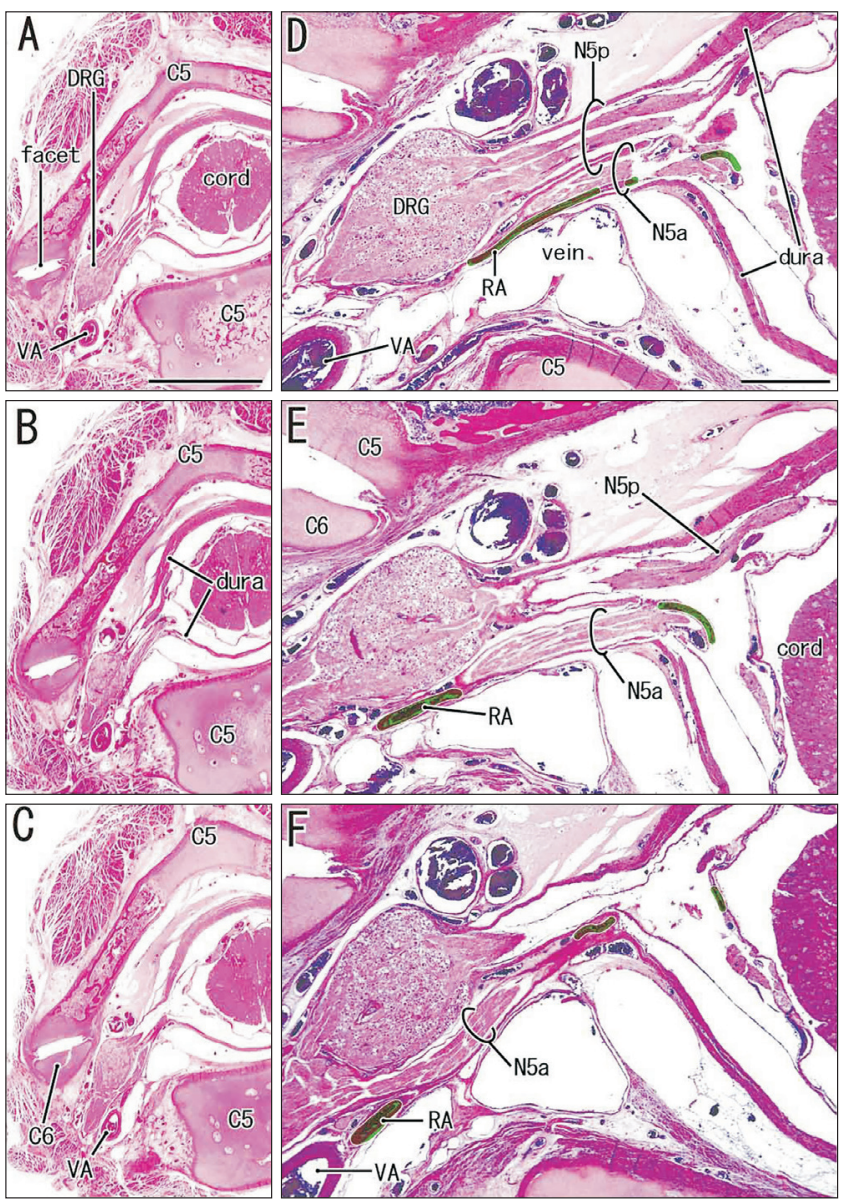

Fig. 6. Horizontal sections including the sixth cervical nerve root. (A, $\mathrm{B}, \mathrm{D}-\mathrm{F}$ ) were prepared at the same magnification (scale bars: $5 \mathrm{~mm}$ in $A, 1 \mathrm{~mm}$ in D). (D-F) are higher-magnification views of the centers of $(\mathrm{A}-\mathrm{C})$, respectively. $(\mathrm{A}, \mathrm{D})$ display a plane $0.2 \mathrm{~mm}$ superior to $(\mathrm{C}, \mathrm{F})$. The anterior nerve root (N6a) as well as two bundles of the posterior root (N6p) take an almost straight course and run in parallel. The radicular artery (RA; colored green) takes a longitudinal course accompanying the anterior root in (D, E). C5, C6, the fifth and sixth cervical vertebrae; DRG, dorsal root ganglion; VA, vertebral artery.

and the features likely reflect the morphology at birth.

In comparison with our previous observations of the thoracolumbar nerve root in near-term fetuses Neural-dural transition at the thoracic and lumbar spinal nerve roots: a histological study of human late-stage fetuses., the cervical nerve root was characterized by a short, straight and lateral course immediately medial to the zygapophysial joint (Table 1). In the thoracolumbar region, a long inferior course was evident in the dural sac because rootlet fasciculation occurred near the spinal cord, and not in the pocket at the nerve exit. Although we had intended to seek structures in the cervical region analogous to those in the thoracolumbar posterior root, i.e., a long tortuous course resembling a string of beads (Fig. 1), no such course was found. Moreover, the distribution of the radicular artery appeared to be sparse in the cervical region. On the other hand, several common features shared by the cervical and thoracolumbar nerve roots were evident, i.e., 1) a pocket-like structure of the dural sac at the nerve exit and 2) a nerve sheath continuous with the dura mater and independently surrounding each of the anterior and posterior roots. Multiple rather than single cervical posterior root bundles appeared to account for the majority of sensory nerve fibers innervating the upper extremity.

The thoracolumbar nerve roots would likely be affected by mechanical stress caused by the marked difference in growth rates between the spinal cord and the vertebral column [3]. Therefore, long nerve roots would be necessary for adaptation to the delayed and marked growth of the vertebral column, leading to development of the cauda equina [2]. In contrast, because of their straight short course and location near the zygapophysial joint, the cervical nerve roots would likely be influenced by movement or dislocation of the vertebrae in children. Cattell and Filtzer [7] reported frequent anterior dislocation of a single cervical vertebra in childhood. Unlike the adult morphology [8], fetal vertebral bodies did not show a saddle-like arrangement providing anteroposterior stability. According to San Román et al. [9], this saddle-like arrangement appears in the late period of childhood.

In a study using horizontal sections from 11 fetuses at 10-24 weeks, Khorooshi et al. [10] reported that the cervical DRG was located at a level lateral to the narrowest part of the interradicular space, in contrast to the situation for thoracic DRG. We agree with their conclusion, with the exception of the DRG of the second cervical nerve. The thoracic DRG is located less than $1 \mathrm{~mm}$ from the dural sac, and therefore it is often adjacent to the dural pocket [3]. In contrast, we found that the cervical DRG was never adjacent to the pocket in frontal sections and the dural sac was not included in sagittal sections showing the cervical DRG. Therefore, the epidural (or subdural) course of the posterior nerve root was long in the cervical (or thoracic) region. In this context, the highly lateral DRG observed here is especially noteworthy. Why was the degree of nerve root fasciculation related to the highly lateral DRG? Growth of the DRG might require some degree of nerve fiber 'bundling' along its course. At the lower lumbar and sacral level, the DRG is likely to exist within the dural sac after the nerve rootlets have shown a long inferior course [2]. Therefore, although it is not critical whether the 

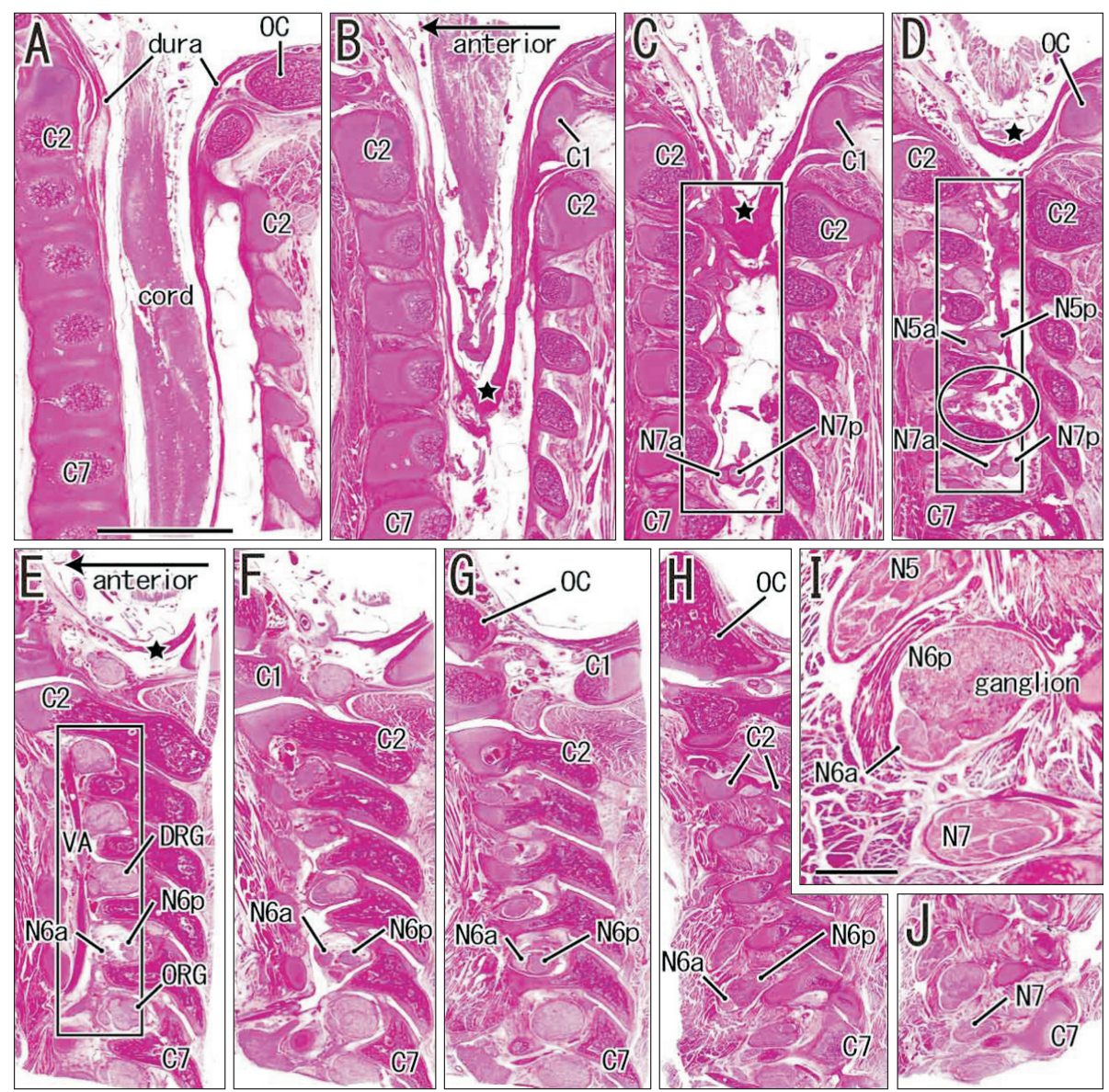

Fig. 7. Abnormality restricted to the sixth cervical nerve: sagittal sections from a fetus with a CRL of $280 \mathrm{~mm}$ (OC29). (A) shows the most medial site in the specimen, and $(\mathrm{I}, \mathrm{J})$ the most lateral. (I) is a higher-magnification view of the central part of $(\mathrm{J})$. (A-H, J) were prepared at the same magnification (scale bars: $5 \mathrm{~mm}$ in A; $1 \mathrm{~mm}$ in I). Stars indicate the bottom of the dural sac. The fifth and seventh cervical rootlets are bundled into two nerve roots (N5a and N5p, N7a and N7p) in (B-D), but no fasciculation is evident in the sixth nerve (circle in D; higher magnification, see Fig. 7). The DRG of the sixth cervical nerve (N6a, N6p) is absent between the vertebral arches (E, F) but appears laterally between the scalenus muscles (I, J). C1, C2, and C7, the first, second and seventh cervical vertebrae; CRL, crown-rump length; OC, occipital basilar part; VA, vertebral artery.

nerve course is subdural or epidural, nerve fiber bundling should occur proximal to the DRG.

Somite development is responsible for segmentation of the spinal nerves [11, 12]. Similarly, the dura mater seemed to be responsible for fasciculation of the spinal nerve roots, as here there was no continuity of the dura with the nerve sheath in unbundled roots with lateral shifting of the DRG. In fact, unilateral segmental anomalies of the vertebrae and ribs in relation to genetic background have been reported [13, 14]. However, the spinal dura shows no segmental border despite its origin from somites $[15,16]$. Therefore, it is unlikely that any unilateral segmental abnormality of the dura would be attributable to redundancy of a specific gene. Conversely, we speculate that any small, regional or transient morphologic feature of the growing dura, such as folding, twisting or tearing, would be unlikely to affect the nerve root sheath, resulting in an unbundled nerve root. This is analogous to wellknown failures of intestinal growth caused by neighboring structures, such as traction or entrapment by the amnion during physiological herniation [17], the peritoneum [18] and vitelline structures $[19,20]$. Traction of the midgut by the leg is also likely [21].

\section{ORCID}

Kei Kitamura: https://orcid.org/0000-0003-3335-1440

Masahito Yamamoto:

https://orcid.org/0000-0001-9683-6678 


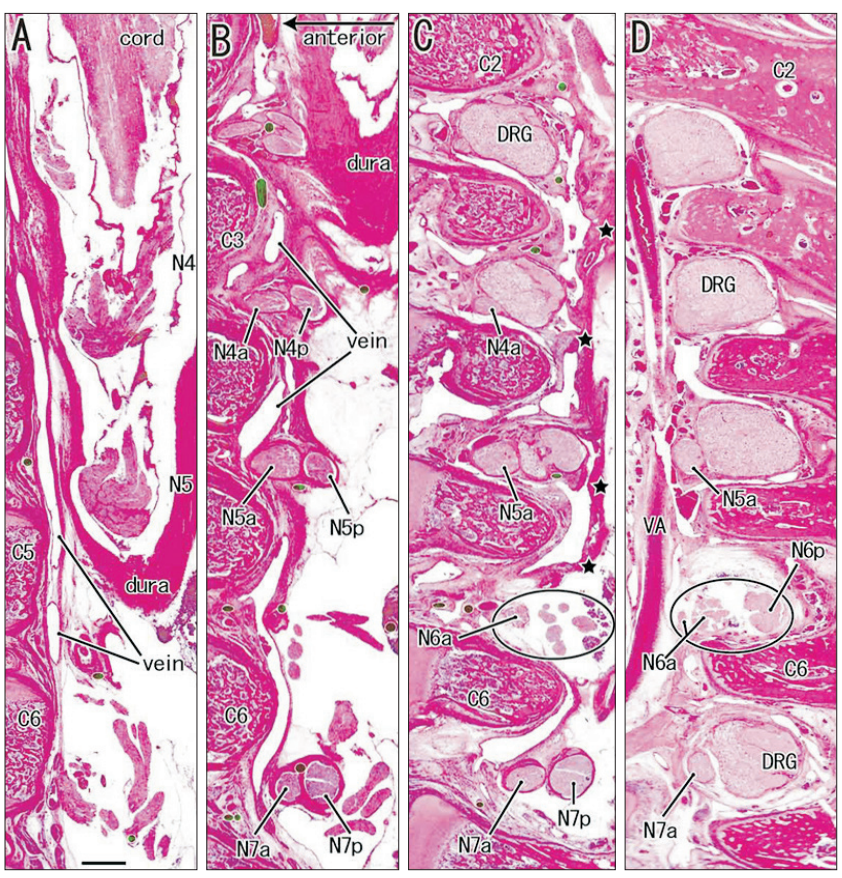

Fig. 8. Abnormality in the sixth cervical nerve: higher magnification. The same specimen as that shown in Fig. 6. (A) shows a plane slightly lateral to Fig. 6B. (B-D) correspond to the squares in Figs. 6C-E, respectively. (A) contains rootlets of the fourth and fifth cervical nerves (N4, N5) in the dural sac. In (B-D), the anterior nerve roots (N4a, N5a, N6a, N7a) can be discriminated from the dorsal root (N4p, N5p, N6p, N7p). The sixth DRG is absent in (D), which includes ganglia at other levels. None of the figures contain radicular artery candidates showing a longitudinal course (colored green). (A-D) were prepared at the same magnification (scale bar in A, $1 \mathrm{~mm}$ ). C2, C3, C5, and C6, the second, third, fifth and sixth cervical vertebrae;

Yoshinosuke Hirota:

https://orcid.org/0000-0001-7131-9252

Noriyuki Sato: https://orcid.org/0000-0002-4508-5568

Toshimasa Machida:

https://orcid.org/0000-0002-3023-0048

Noboru Ishikawa: https://orcid.org/0000-0002-8207-2213

Hitoshi Yamamoto:

https://orcid.org/0000-0002-4181-6271

Gen Murakami: https://orcid.org/0000-0001-7181-5475

Shinichi Abe: https://orcid.org/0000-0003-4632-9736

\section{Author Contributions}

Conceptualization: GM. Data acquisition: KK, MY, YH, NS, TM. Data analysis or interpretation: KK, NI. Drafting of the manuscript: HY, GM. Critical revision of the manuscript: SA. Approval of the final version of the manuscript: all authors.
Table 1. A comparison between cervical and thoracolumbar nerve roots

\begin{tabular}{|c|c|c|}
\hline & Cervical & Thoracolumbar \\
\hline $\begin{array}{l}\text { Angle of nerve root course at } \\
\text { the exit from the dural sac }\end{array}$ & $90^{\circ}-120^{\circ}$ & $120^{\circ}$ or more \\
\hline $\begin{array}{l}\text { Pockets of the dural sac at the } \\
\text { exit of nerve root }\end{array}$ & + & + \\
\hline $\begin{array}{l}\text { Independent sheathes for the } \\
\text { posterior and anterior roots }\end{array}$ & + & + \\
\hline $\begin{array}{l}\text { A highly tortuous course of } \\
\text { the posterior root }\end{array}$ & - & + \\
\hline $\begin{array}{l}\text { Numbers of bundles of the } \\
\text { posterior root }\end{array}$ & 2 or 3 & 1 \\
\hline $\begin{array}{l}\text { Fasciculation of nerve } \\
\text { rootlets }\end{array}$ & In the pocket & Proximal to the pocket \\
\hline Course of nerve roots & lateral, short & inferior, long \\
\hline $\begin{array}{l}\text { Segmental abnormality in } \\
\text { bundling }\end{array}$ & likely & no reports \\
\hline Site of the DRG & near facet & near rib head \\
\hline
\end{tabular}

DRG, dorsal root ganglion.

\section{Conflicts of Interest}

No potential conflict of interest relevant to this article was reported.

\section{Acknowledgements}

We are grateful to Prof. Dr. Jose Francisco RodríguezVázquez (Department of Anatomy, Universidad Complutense) for allowing us to use his materials.

\section{References}

1. Tubbs RS, Lobashevsky A, Oakes P, D'Antoni AV, Hattab E, Topp K, Loukas M, Spinner R. Meningeal relationships to the spinal nerves and rootlets: a gross, histological, and radiological study with application to intradural extramedullary spinal tumors. Childs Nerv Syst 2015;31:675-81.

2. Jang HS, Cho KH, Chang H, Jin ZW, Rodriguez-Vazquez JF, Murakami G. The filum terminale revisited: a histological study in human fetuses. Pediatr Neurosurg 2016;51:9-19.

3. Cho KH, Jin ZW, Abe H, Shibata S, Murakami G, RodríguezVázquez JF. Neural-dural transition at the thoracic and lumbar spinal nerve roots: a histological study of human late-stage fetuses. Biomed Res Int 2016;2016:8163519.

4. Yi M, Lee JW, Yeom JS, Joe E, Hong SH, Lee GY, Kang HS. C2 nerve root on magnetic resonance imaging of occipital neuralgia. Spine (Phila Pa 1976) 2014;39:1077-83.

5. O'Rahilly R, Muller F, Meyer DB. The human vertebral column at the end of the embryonic period proper. 1 . The column as a whole. J Anat 1980;131(Pt 3):565-75.

6. Castellana C, Kósa F. Morphology of the cervical vertebrae in 
the fetal-neonatal human skeleton. J Anat 1999;194(Pt 1):147-52.

7. Cattell HS, Filtzer DL. Pseudosubluxation and other normal variations in the cervical spine in children. A study of one hundred and sixty children. J Bone Joint Surg Am 1965;47:1295309.

8. Bogduk N. Functional anatomy of the spine. Handb Clin Neurol 2016;136:675-88.

9. San Román P, Palma JC, Oteo MD, Nevado E. Skeletal maturation determined by cervical vertebrae development. Eur J Orthod 2002;24:303-11.

10. Khorooshi MH, Fischer Hansen B, Keeling J, Nolting D, Kjaer I. Prenatal localization of the dorsal root ganglion in different segments of the normal human vertebral column. Spine (Phila Pa 1976) 2001;26:1-5.

11. Müller F, O'Rahilly R. Somitic-vertebral correlation and vertebral levels in the human embryo. Am J Anat 1986;177:3-19.

12. Müller F, O'Rahilly R. Segmentation in staged human embryos: the occipitocervical region revisited. J Anat 2003;203:297-315.

13. Hashimoto J, Murakami G, Tsugane MH, Chisaka O, Capecchi MR, Ogino T. Lumbosacral plexus in Hoxa9 knockout mice with special reference to their nerve variations identified according to whether they were interphenotypic or intergenotypic differences. Kaibogaku Zasshi 1999;74:609-30.
14. Bots J, Wijnaendts LC, Delen S, Van Dongen S, Heikinheimo K, Galis F. Analysis of cervical ribs in a series of human fetuses. J Anat 2011;219:403-9.

15. Klika E, Zajícová A. The development of meninges in chicken embryos. Anat Anz 1976;140:379-86.

16. O'Rahilly R, Müller F. The meninges in human development. J Neuropathol Exp Neurol 1986;45:588-608.

17. Hunter AG, Seaver LH, Stevenson RE. Limb-body wall defect. Is there a defensible hypothesis and can it explain all the associated anomalies? Am J Med Genet A 2011;155A:2045-59.

18. Raphaeli T, Parimi C, Mattix K, Javid PJ. Acute colonic obstruction from Ladd bands: a unique complication from intestinal malrotation. J Pediatr Surg 2010;45:630-1.

19. Komuro H, Hoshino N, Urita Y, Fujishiro J, Sakamoto N, Ono K, Kaneko M. Pathogenic implications of remnant vitelline structures in gastroschisis. J Pediatr Surg 2010;45:2025-9.

20. Fukuzawa R, Toma M, Nomura A. Histology of a paraumbilical band in a neonate with gastroschisis. Pediatr Dev Pathol 2011;14:493-5.

21. Kim JH, Hwang SE, Rodríguez-Vázquez JF, Murakami G, Cho BH. Liver agenesis with omphalocele: a report of two human embryos using serial histological sections. Pediatr Dev Pathol 2014;17:431-40. 